\title{
In-situ calibration experimental method for Infrared thermography applied to the heat transfer analysis for composite parts during manufacturing based on Resin Infusion technique
}

\author{
by U. Pineda*, N. Montés* ,F. Sánchez*, F. Bensadoun** and E. Ruiz**
}

*Department of Building and Industrial Production, CEU Cardenal Herrera University, C/ San Bartolomé, 55 46115 Alfara del Patriarca, Spain, upineda@uch.ceu.es, nimonsan@uch.ceu.es fernando.sanchez@uch.ceu.es

** Chair on Composites of High Performance (CCHP), École Polytechnique de Montréal, Department of Mechanical Engineering, P.O. Box 6079, Station "Centre-Ville", Montreal, Canada, H3C 3A7, Research Centre on Plastics and Composites (CREPEC), edu.ruiz@polymtl.ca, farida.bensadoun@polymtl.ca

\begin{abstract}
This work purposes an in-situ calibration experimental method based on Infrared thermography combined to proper heat flux sensors in order to analyze the curing stage in the manufacturing of thermoset composite parts. This method can provide successfully bidimensional data on areas wherever monitoring, measuring or analyzing the thermal transient behavior as well as compute temperature and heat flux gradients. This could be very interesting at the industrial level for large composite parts since can be obtained a greatest monitoring capability, measurement and heat transfer analysis than just by means of use zero-dimensional sensors. Finally, it presents the calibration algorithms to measure the instantaneous heat flux released by a composite part from anywhere itself during its manufacturing.
\end{abstract}

\section{Introduction}

Liquid Composite Moulding processes (LCM) are widely used to produce composite parts. They include various techniques like Resin Transfer Moulding (RTM), Vacuum Assisted Resin Infusion (VARI), Vacuum Assisted Resin Transfer Moulding (VARTM), Resin Infusion (RI), etc. In particular, Resin Infusion (RI) manufacturing technique is used for its ability to produce large components with a good quality/cost rate in limited series [1]. This technique uses vacuum pressure to drive the thermoset resin into a fibrous laminate. The fiber preform is laid dry into the mould and vacuum is applied before the uncured thermoset resin is introduced [2]. Fig 1 presents the steps of the Resin Infusion process from preforming to demoulding. It has to be noted that the countermould can be made of a flexible material, thus reducing costs allowing manufacturing pieces of practically any size [3]. The flexible countermould is usually a transparent bagging film in strong thermal contact with the materials processed inside the mould due to the vacuum pressure used. After the resin injection, due to the addition of catalyst, the cross-linking is accelerated and the viscosity increases which means that the thermoset resin is in-curing stage into the mould [4]. Consequently, mould releases heat into the environment while fiber reinforcement and cured thermoset become a single rigid part.

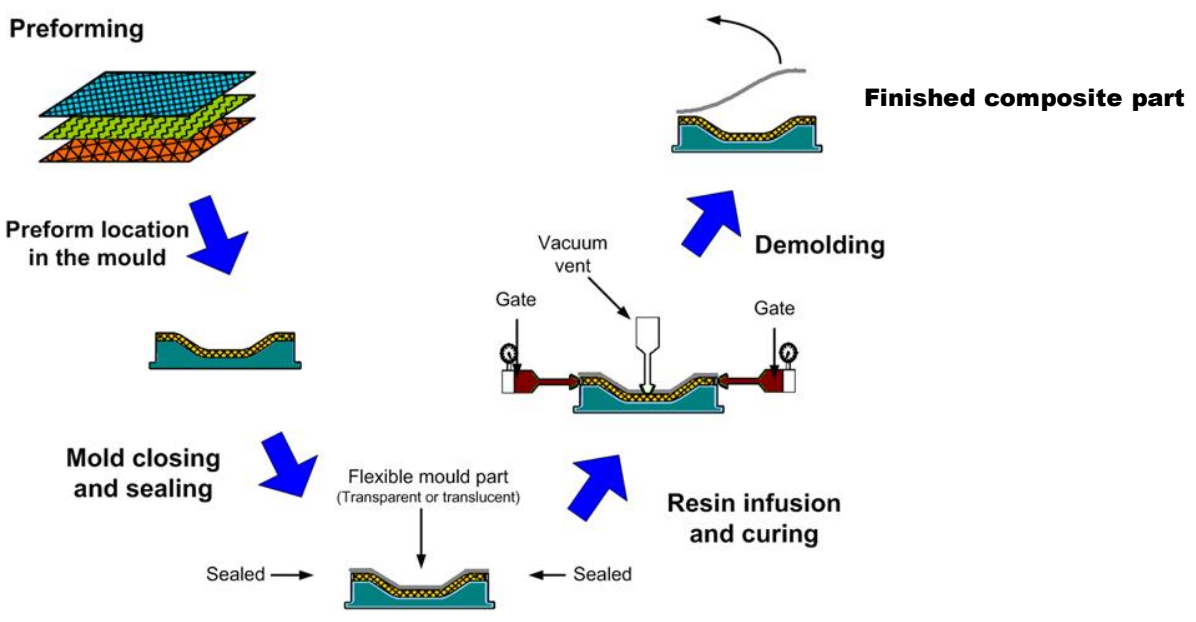

Fig 1 Resin Infusion manufacturing Process

At present this manufacturing process still presents reliability challenges for industrial production [1]. Some of them are the thickness and the fiber volume fraction of the final piece are not well controlled, first because of the use of a vacuum bag instead of a rigid mold and second due the large variation of the preform volume when vacuum and pressure are applied [5]. Moreover, it lacks a good understanding of the cure of moulds because of their low thermal conductivity and the heat of reaction released during the polymerization and their relationship with the not well-controlled parameters 
abovementioned. Then, heat released by moulds during curing stage appears to be interesting to analyze since this can lead to significant thermal gradients and temperature peaks depending on the part geometry, which ultimately could generate residual stresses, and thus polymer degradation. Residual stresses have a significant effect on the part quality and mechanical properties, generating warpage or initiating stress concentration in the matrix and delamination of the composite part [6]. Then, processing temperatures needs to be optimally controlled so that thermal gradients remain small and the optimized mechanical properties can thus be obtained for the finished parts.

Currently, one of the most common manufacturing practices is that curing stage draws heavily on experience. This means that the predictability and consistency of production depend largely on experts [1]. Usually, the experts design the curing cycles to be applied to moulds based on the resin manufacturer specifications, their experience and thermal characterizations of materials. To characterize the polymerization of thermoset resins, Differential Scanning Technique (DSC) is commonly used [7]. This technique allows the monitoring of the cure kinetics of by measuring of the heat flux generated during a chemical reaction. This is extensively used in the determination of cure and post-cure parameters. One of the most important parameter which is obtained by DSC is the total energy of reaction from a small and activated neat resin sample (just a few milligrams), and other interesting parameters as the proper work temperatures for the resin processing, the reaction rate, and the degree of cure (DoC), etc. On top of the mentioned statement, an accurate kinetic model is needed in order to predict the optimal thermal cycle for composite parts manufacturing.

\section{Heat Transfer Measurement Techniques for moulds in the curing stage}

In thermosets matrices, if a large amount of resin is used (a few kilograms), a mass effect has to be considered for the cure kinetics calculation. For DSC testing, due to the scale of the sample, only small amount of resin can be studied and thus the mass effect is not visible. In this way, non-homogeneity of thickness along fiber laminate as well as changes of manufacturing conditions and resin handling according to manufacturer specifications may generate different curing behavior [8]. Therefore, if some places of the mould present actual temperatures and heat fluxes which deviate from the predictions regarding its cure kinetics, this may prove a lack of accurate knowledge on the polymerization of the mould. Of course an accurate response is also affected by the reliability and efficiency of the sensing technique for the heat transfer. The reliability and efficiency of detecting the heat fluxes from the any place of the mould while they cure can be improved according to the geometric shape, dimension and location of the sensors used. However, these characteristics are also the disadvantage compared to alternative methods of sensing described below.

Several methods, intrusive and non intrusive, exist for measuring and analyzing the polymerization of local areas during cure process, according to literature review. Intrusive methods are distinguished by contact between surfaces of both mould and sensor. In contrast non-intrusive not require physical contact on the mould. To measure the curing of mould, both detection techniques exploit changes in the physical properties of the thermoset resin. For instance, contact sensors take advantage of changes of optoelectronic properties [9-11], electrical properties [12-14], acoustic properties [15-17], and thermal properties [7;18-21]. Meanwhile, non-contact techniques exploit mainly acoustic properties [22] and thermal properties [23]. Thus, non-contact sensors have been explored a lot less than contact sensors. In this work, a method that investigates the temperature and heat flux changes during the curing stage for a vinylester-based composite will be presented. It is a common manufacturing practice placing zero-dimensional thermal sensors to measure the heat flux or temperature [24]. However, sensor locations are often defined by expert criteria, which they may be not enough for the cure monitoring of the studied mould.

Recent publications [25-27] mentioned the use of the thermal camera calibrated by in situ method. They showed that the temperature is the key parameter for the calibration and data measurement. Moreover, none of these publications has been applied to composites manufacturing. In this work, the calibration of the temperature is done instantaneously, and it is used as a reference for computing the total heat flux which is a key for curing characterization of composites. According to literature, this study is the first of its kind for in-situ calibration done by thermography applied during the live curing process (exothermic reaction) of a composite part. Abovementioned, heat flux measurement

Therefore, our work focuses on measuring the heat flux and temperature by contact and non-contact sensing during an exothermal heat process due to on-going polymerization of a mould. This proposes an in-situ calibration experimental technique for heat flux measuring that is released from anywhere mould during the exothermic curing process, instantaneously. This method offers flexibility and versatility to measure heat flux any location on the mould, at the same instant of time. Mentioned capacities are obtained by combining the accuracy of zero-dimensional sensors and the bidimensional detection of infrared thermography, together.

\section{Experimental Setup for development of an in-situ calibration technique}

Fig 2 and Fig 3 show the setup for the manufacturing of a composite part which is a square shape $30 \times 30 \mathrm{~cm}^{2}$ plate by $\mathrm{RI}$ technique where the resin inlet is at the left side and the outlet at the right end side. The laboratory temperature is controlled at $22^{\circ} \mathrm{C}$ and humidity at $50 \%$. The reinforcement used in this test was $0^{\circ} / 90^{\circ}$ woven fibreglass fabric and vinylester thermoset resin. The mould shown (Fig 2) is placed inside a black box as shown in Fig 3 -on the right. The lower mould is made of a glass plate and the countermould a plastic bagging that is flexible and transparent with an elongation of break $400 \%$. The catalysed liquid resin is injected into the mould through the inlet pipe (filling stage) 
until it fills completely. At that time, the resin feed to the mould is cut completely and curing stage will start when a short time elapses.

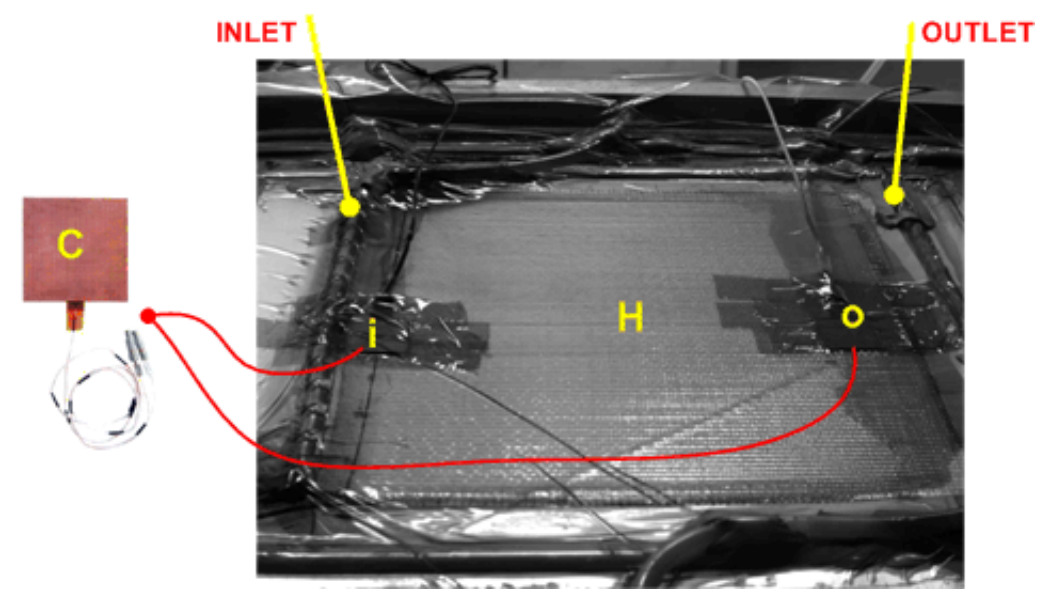

Fig 2 Setup for Composite manufacturing of plates by Resin Infusion technique. Sensors (i) and (o) are corresponding to the near inlet and outlet Heat Flux-Temperature sensor. (C) is the contact Heat Flux-Temperature Sensor used
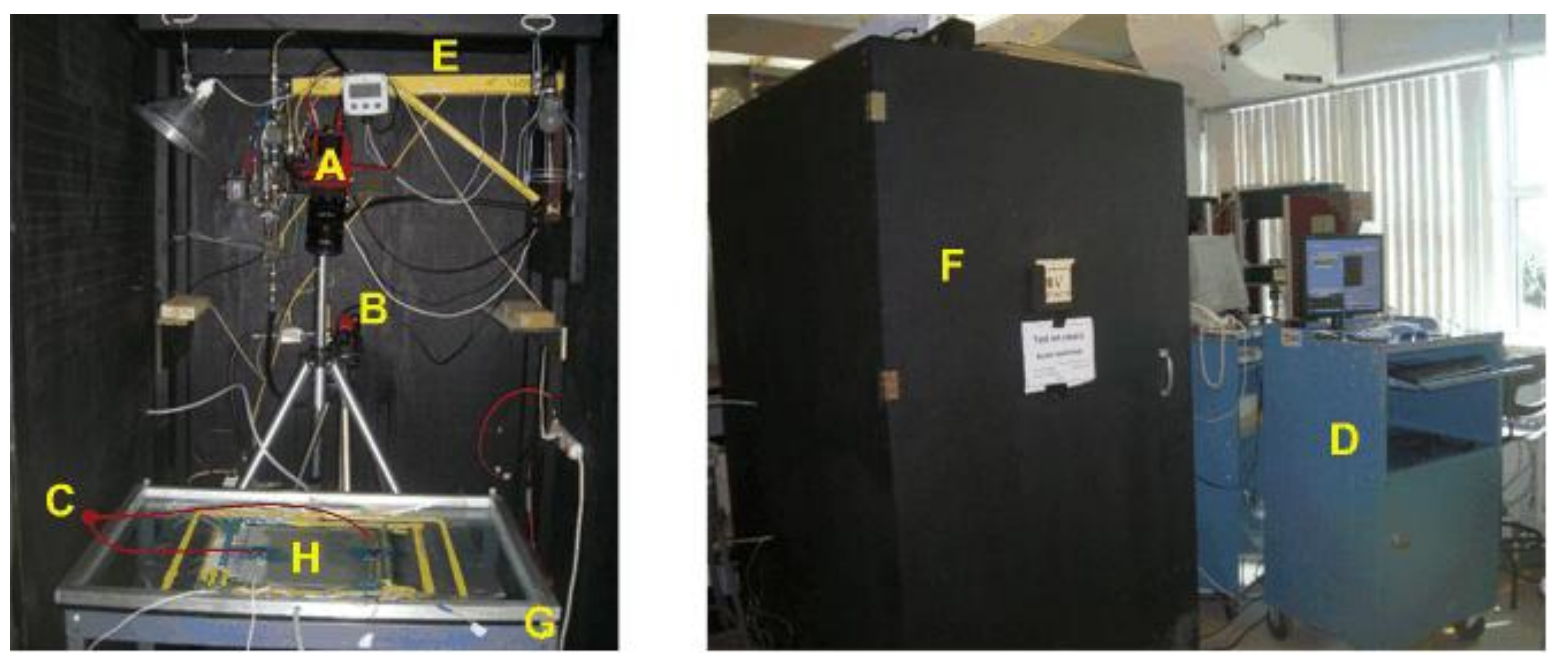

Fig 3 On the Left, location of $(\boldsymbol{H})$ mould for square part to be manufactured on the (G) table which is sensed by means of $(\boldsymbol{A})$ thermal camera, $(\boldsymbol{B})$ visible camera, $(\boldsymbol{C})$ contact Heat Flux-Temperature sensors and $(\boldsymbol{E})$ surroundings temperatures sensor. Moreover, (F) is mean, black box is opened. On the Right, $(\boldsymbol{F})$ is closed which is made of wood for the thermal-protection to experimentation that avoids convection, sudden thermal disturbs into the tests, and finally, (D) is the acquisition system by computer.

A custom acquisition software based on graphical programming LabView 8.6 was developed in a previous study [24] and will be used as a reference in this work. This data acquisition system uses thermal sensors to retrieve the heat flux during polymerization. This system receives the thermal information needed for the thermal camera calibration. These data come from the mould while this presents thermal changes and flow front changes in the filling stage. These changes are captured instantaneous and simultaneously. In Fig 2 and Fig 3 on the left side, near to the inlet and vent, a pair (Ci and Co) of contact sensor is placed for local heat flux and temperature measuring; a thermal camera is vertically separated 1.3 meters above the mould for the thermal viewing. The manufacturers of the "thermo-sensitive" sensors are thermoflux and Captec. During the experiments, all the equipment is placed inside the black box (see Fig 3 on the right) in order to prevent that external interactions, not-controlled heat sources or thermal noise which may affect the thermal camera (TVS-500EXZ manufactured by NEC) as also the contact sensors while exothermic heat is generated by polymerization of the mould. 


\section{Experimentation}

\subsection{Qualitative thermal evolution through historical thermography for a composite part during curing}

Table 1 Qualitative evolution of temperatures by thermal images during curing stage of the square plate
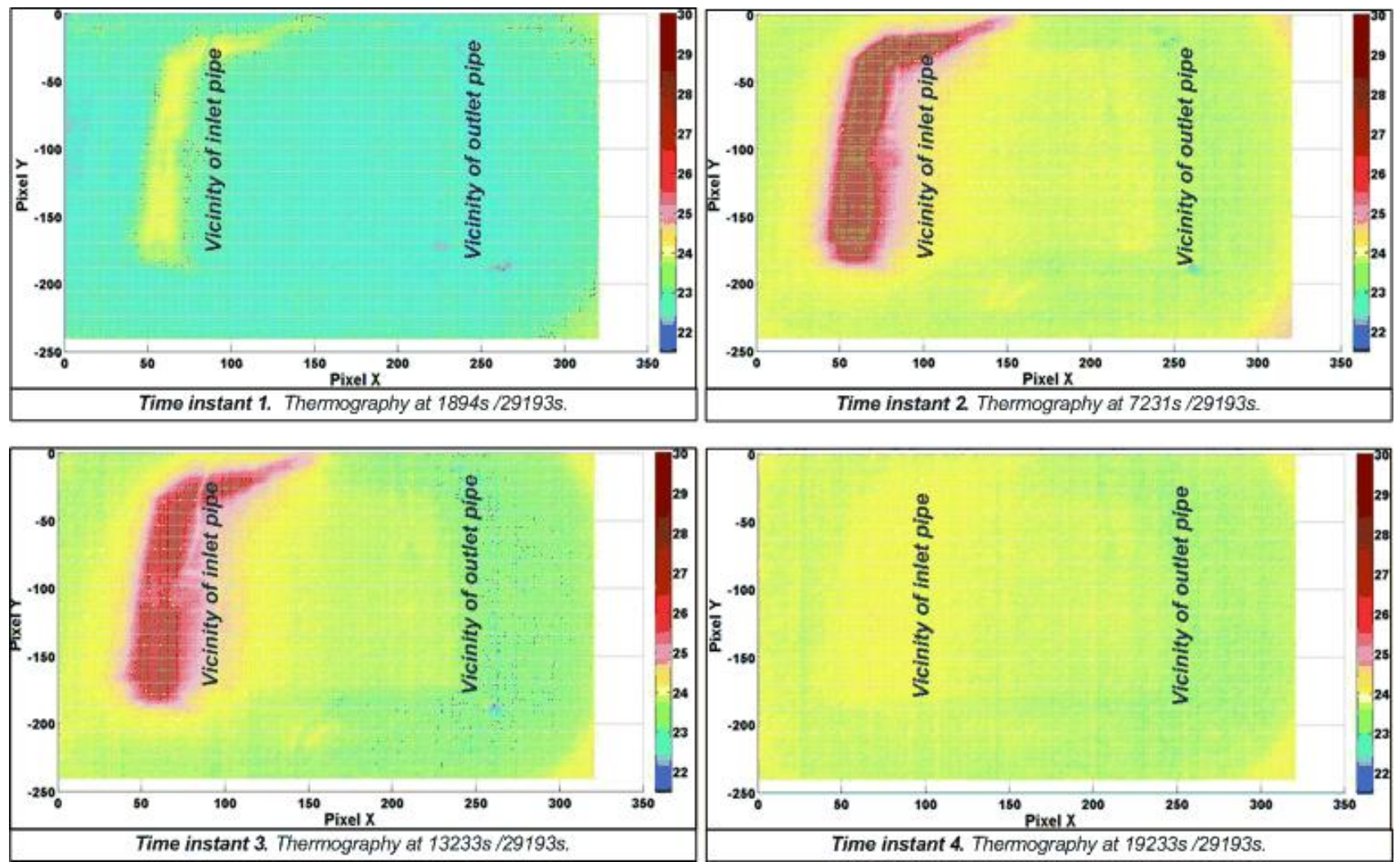

Qualitatively, thermal images show the heat transfer during curing stage, experienced this by the mould due its exothermicity caused by the polymerization, as can be seen in Table 1 . For instance, the Table 1 in the time instant 1 shows how regions close to the resin injection tubing increase temperatures because of a mass concentration around inlet is higher than for areas near at the outlet. This thermal variation is also shown in the Fig 5 through the plots of thermal signals, $Q \boldsymbol{i}_{c}$ and $\Delta \boldsymbol{T} \boldsymbol{i}_{c}$. Thermal imaging allows direct calculation of apparent temperatures for each pixel using the algorithm published by NEC, the manufacturer of the thermal imaging camera [28]. Further, There have already been published works which propose methods of calibration in order to enable the thermography quantitatively, as [25].

These apparent temperatures are not accurate because of uncertainty in the value of the emissivity of the surface (it is unknown to the plastic film used as a countermould in this test) that is non-constant when the measurement surface is subjected to thermal transients [29] or exothermic processes as reported [30]. In [30] developed a method to measure true temperature of an exothermic process caused by resin curing by means of a radiometer based on fiber optic, not in thermal image. Additionally, the publication [31] are explained quite well the reasons for uncertainty in temperature measurement caused by the emissivity. Consequently, measurements based on the radiation capture such as the thermography have to be calibrated by means of thermal information acquired by other sensors (reliable and accurate), simultaneously. The quantitative thermography and experimentation are discussed below in section 5.2

\subsection{Quantitative Thermography of test. Comparative study by conductive and radiative sensors for different mould places.}

The data acquisition of the polymerization process starts as soon as the mould starts to be filled with resin. The heat release is closely monitored for several hours $(\approx 8$ hours) until the polymerization is completed. This is when the mould temperatures show to be equal to room temperature. The sampling rate is an acquisition/second, simultaneously from all sensors. Heat flux and temperature contact sensors, $(\boldsymbol{C i})$ and $(\mathbf{C o})$ are located as shown Fig 2 and Fig 4 . Thus, as it evolves mould exothermic process, contact temperature and heat flux both in the inlet $\left(\boldsymbol{T i}_{c}, \boldsymbol{Q i} \boldsymbol{i}_{c}\right)$ as in the outlet $\left(\boldsymbol{T o}_{\boldsymbol{c}}\right.$, $\mathbf{Q o}_{\mathbf{c}}$ ) are measured instantaneously. Last contact signals mentioned are the in-situ thermal reference to calibrate the apparent temperatures measured by thermal camera, whether they are located in the inlet $\left(\boldsymbol{T i}_{n c}\right)$, outlet $\left(\boldsymbol{T o}_{n c}\right)$ or other place $\left(T p_{n c}\right)$ along the mold (see Fig 4 and Fig 7 ). Therefore, by the contact sensors as in-situ reference, each pixel can offer an approximated heat flux measurement of the process. 


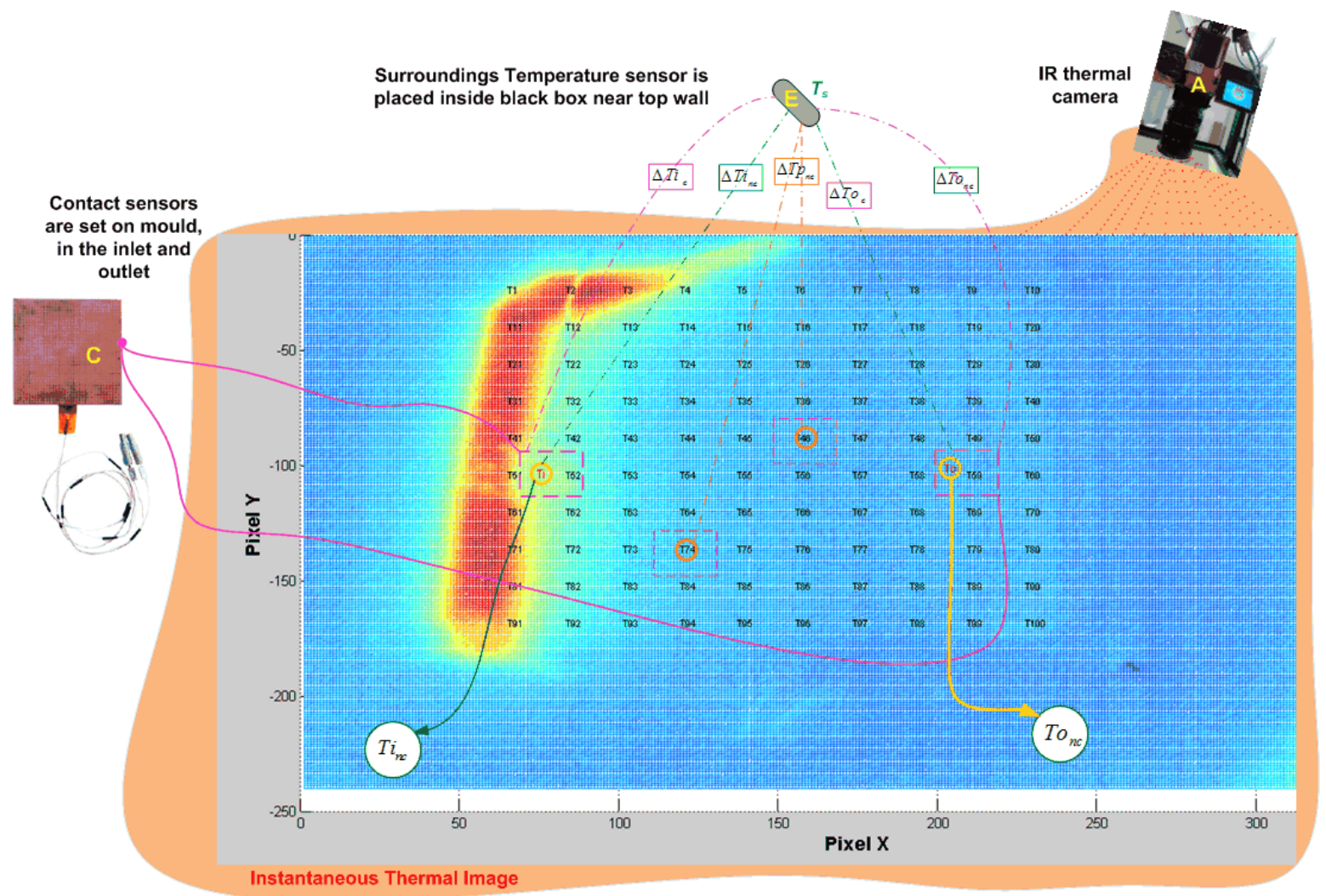

Fig 4 Right, analysis process of thermography (time instant 5231/29193s or image instant 16011/39971) and left, Contact Heat Flux-Temperature Sensor gives accuracy measurements. Magenta dashed lines are the square projection on the thermography image to give relative location idea of inlet and outlet sensors at thermal images.

The surroundings temperature $T_{s}$ throughout the time range remains almost constant with a variation of about 22 to $22.5^{\circ} \mathrm{C}$, guaranteeing quasi-isothermal conditions. Therefore, the temperature differences $(\Delta \boldsymbol{T})$ are computed by subtraction as, $\Delta \boldsymbol{T}=\boldsymbol{T}-\boldsymbol{T}_{\boldsymbol{s}}$, where $\boldsymbol{T}$ can be contact or non-contact temperature. In Fig 5 it is show $\Delta \boldsymbol{T} \boldsymbol{i}_{\boldsymbol{c}}$ and $\Delta \boldsymbol{T} \boldsymbol{o}_{\boldsymbol{c}}$ for the $\boldsymbol{T i}_{\boldsymbol{c}}$ and $\boldsymbol{T o}_{\boldsymbol{c}}$ respectively. As also shown the Fig $5, \Delta \boldsymbol{T i}_{\boldsymbol{c}}$ and $\Delta \boldsymbol{T} \boldsymbol{o}_{\boldsymbol{c}}$ as well as $\boldsymbol{Q i}_{\boldsymbol{c}}$ and $\mathbf{Q o}_{\boldsymbol{c}}$. are different. These differences are related to the locations on the mould where sensors monitors the heat flux released. Near to the inlet tubing is noted a higher mass concentration of resin than other places along the mould which causes a difference in thickness that is slightly greater at the inlet than at the outlet. The latter implies that the heat released from the mould is not homogeneous along itself therefore the finished part will have thickness variations along itself.

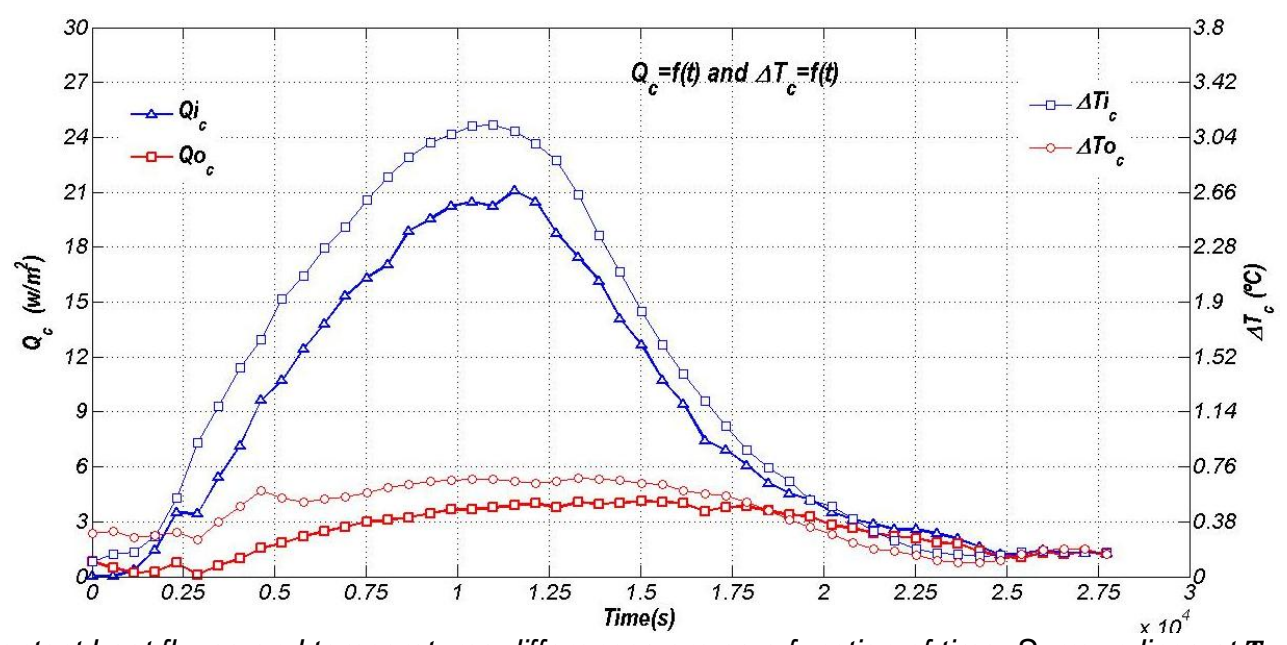

Fig 5 Contact heat fluxes and temperatures differences are as a function of time. Surroundings at $\boldsymbol{T}_{\boldsymbol{s}} \cong \mathbf{2 2}^{\circ} \mathrm{C}$.

In the Fig 5 and Fig 6 , the temperature differences $(\Delta \boldsymbol{T})$ are computed by subtraction as, $\Delta \boldsymbol{T}=\boldsymbol{T}-\boldsymbol{T}_{\boldsymbol{s}}$, where $T$ can be contact or non-contact temperature. By these figures can be confirmed that while heat fluxes on the mould ( $\boldsymbol{Q}_{c}$ ) and $\Delta \boldsymbol{T}_{c}$ behaves similarly, either the inlet or outlet. In this sense, calculations of $\Delta \boldsymbol{T}_{c}$ maintain proportionality with $\boldsymbol{Q}_{c}$ as a 
function of time (see Fig 5). Likewise, $\boldsymbol{Q}_{c}$ has a proportional behavior with $\Delta \boldsymbol{T}_{n c}$. This means that $\boldsymbol{Q}_{c} \boldsymbol{\alpha} \Delta \boldsymbol{T}_{c}$ as $\boldsymbol{Q}_{c} \boldsymbol{\alpha} \Delta \boldsymbol{T}_{n c}$ but is needed to check the why contact and non-contact responses do not match as they observe in the Fig 6 . Then, assuming that the contact sensors data are accurate and error-free, in the Fig 6, it can be see two kinds of deviations in the curves. Firstly, the plots of $\boldsymbol{Q}_{c}=\mathbf{f}\left(\Delta \boldsymbol{T}_{n c}\right)$ show a horizontal shift respect to the plots of $\boldsymbol{Q}_{c}=\boldsymbol{f}\left(\Delta \boldsymbol{T}_{c}\right)$. Secondly, they both go up and down by different thermal paths. That deviations, point by point can be corrected knowing the causes that produce them. Then, abovementioned deviations must be analyzed as follow below:

$Q_{c}=f\left(\Delta T p_{n c}\right)$ and $Q_{c}=f\left(\Delta T_{c}\right)$ have a horizontal shift. The induced error through the measured temperatures by thermal camera is evident here in the Fig 6. By infrared thermography is not possible to measure the accurate temperatures but apparent temperatures. Therefore, instantaneous thermal image must be calibrated through the accurate contact sensors thus obtaining $\boldsymbol{Q}_{c}=\boldsymbol{f}\left(\Delta \boldsymbol{T}_{n c \text {, corr }}\right)$ or $\boldsymbol{Q}_{c}=\boldsymbol{f}\left(\Delta \boldsymbol{T} \boldsymbol{p}_{n c}\right)$ whether is applied this to any pixel. For the calibration mentioned is needed to compute the error at each time instant as $\mathbf{e}(\boldsymbol{t})=\boldsymbol{T}_{c}(t)-\boldsymbol{T} \boldsymbol{p}_{n c}(\boldsymbol{t})$, where, $\mathbf{e}(\boldsymbol{t})$ is the error $\boldsymbol{T}_{c}(\boldsymbol{t})$ is the contact temperature on the mould and $\boldsymbol{T} \boldsymbol{p}_{n c}(\boldsymbol{t})$ is the pixel's apparent temperature.
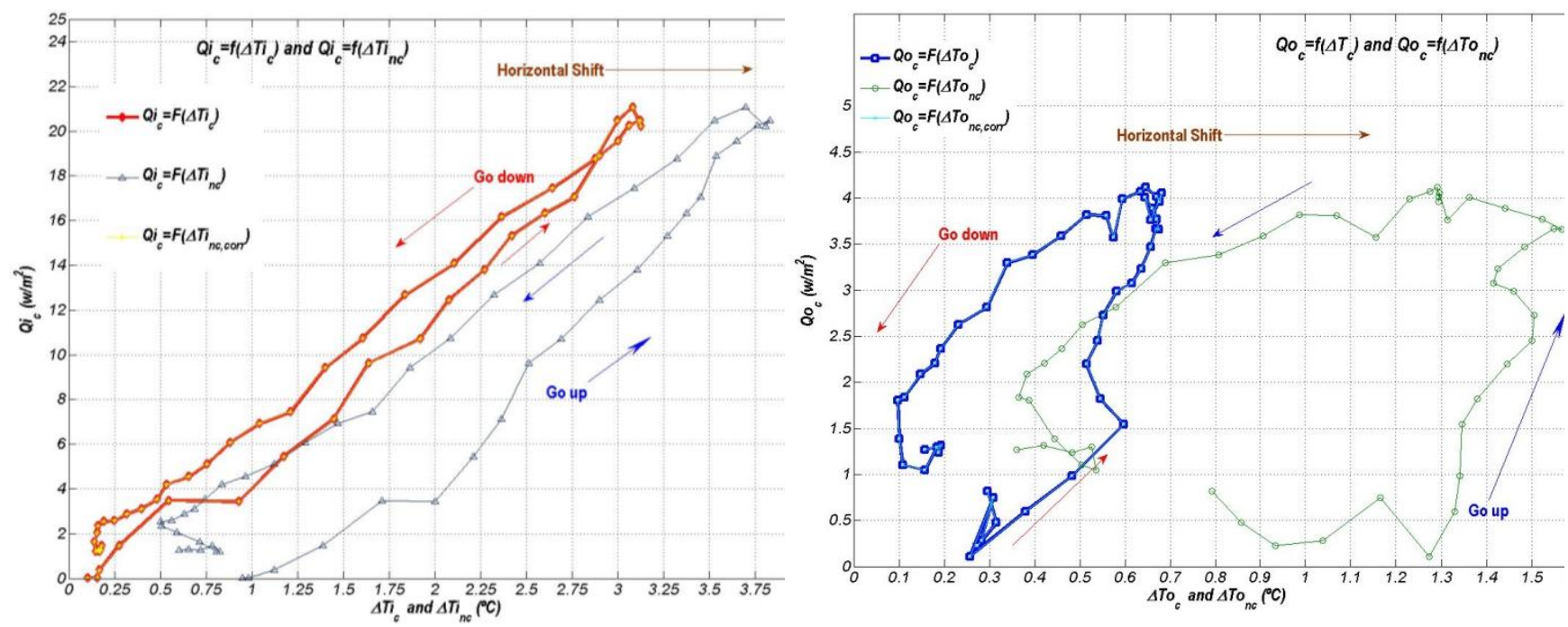

Fig 6 Contact Heat Flux as a function of temperature differences by contact and non-contact. On the Left, inlet behavior. On the right, outlet behavior

$Q_{c}=f\left(\Delta T p_{n c}\right)$ and $Q_{c}=f\left(\Delta T_{c}\right)$ describe different thermal paths for going up and down. Particularly natural convection effects cause it. The analysis is the following: the Fig 5 shows that the mould releases heat into the surroundings during the initial time of $11500 \mathrm{~s}$ where $\boldsymbol{Q}_{c}$ is a portion of all heat released by the mould. Thus, the released heat $\boldsymbol{Q}_{c}$ is moved by natural convection to the mould's surroundings so there exists a small heat transfer between the mould and the interior walls of the black box. In the Fig 9 a small increase $\mathrm{h}$ before and after the exothermal heat peak confirms the natural convection. After the exothermal peak, as shown in Fig 5 and Fig 9 , heat flux $\boldsymbol{Q}_{c}$ decreases up to almost reach thermal balance and $\mathrm{h}$ increases just before reaching the thermal equilibrium . In fact, $\boldsymbol{Q}_{c}=\boldsymbol{f}\left(\Delta \boldsymbol{T} \boldsymbol{p}_{n c}\right)$ and $\boldsymbol{Q}_{c}=f\left(\Delta \boldsymbol{T}_{c}\right)$ go down through different paths as they go up (see Fig 6).

\section{In situ calibration experimental method for infrared thermography}

The flow chart diagram in the Fig 7 shows the algorithm that allows heat flux to be computed for each pixel which leads to this method to append the capacity to visualize heat thermal images instead temperature. It takes the work done in [32] as main reference to compute $h$.

The behavior shown in the Fig 6 is due to the natural convection and it becomes further evident when curves go down, because the factor $h$ increases from the exothermal heat peak up to the times at the end of exothermal process in the Fig 9. Moreover, the factor $h$ almost no change and is recurring for wherever on the mould as a function of time. This proportionality factor $h$ or convection factor can be computed as follow, $h(t)=Q_{c}(t) / \Delta T p_{n c}(t)$. Where, $Q_{c}(t)$ is the contact heat flux delivered by sensors on the mould and $\Delta T p_{n c}(t)$ is the temperature differences between calibrated thermal image and $\boldsymbol{T}_{s}(t)$. Therefore, attempting to explain this kind of exothermal heat process by means of only one fit straight line or a just factor of proportionality for all time domain is not suitable since the kind of inaccuracy that presents this thermal behavior must be updated under time in order to obtain better results. Finally, it can be compute the calibrated heat flux image according to algorithm that shown the Fig 7.

In the Fig 8 is observed the error evolution that is very low and close to null and almost of the same order of magnitude for wherever on the mould. 


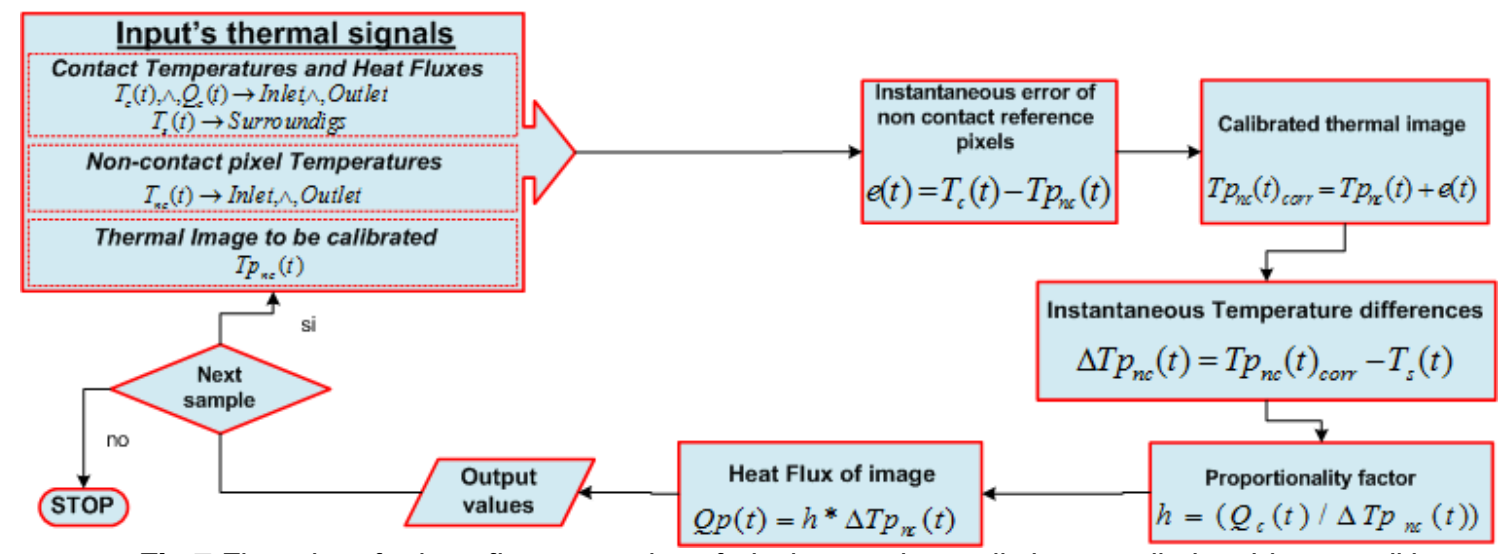

Fig 7 Flow chart for heat flux computing of pixels experimentally in controlled ambient conditions

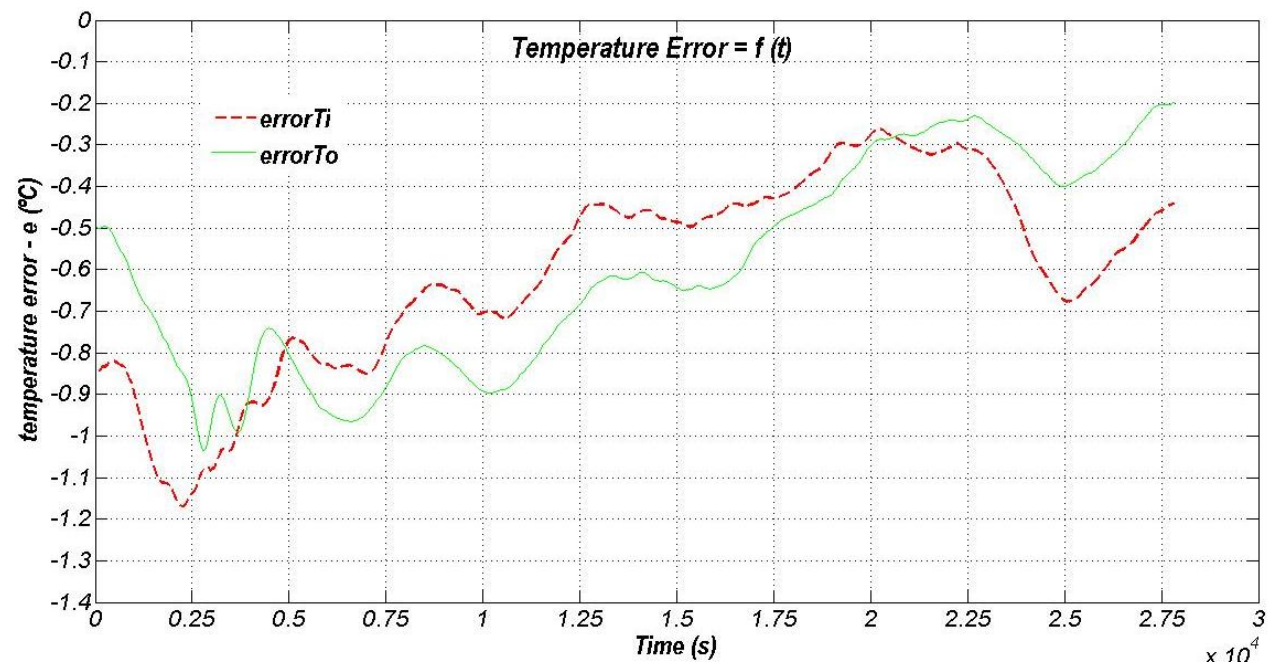

Fig 8 Instantaneous Temperature Error (Camera temperatures vs. Contact temperatures)

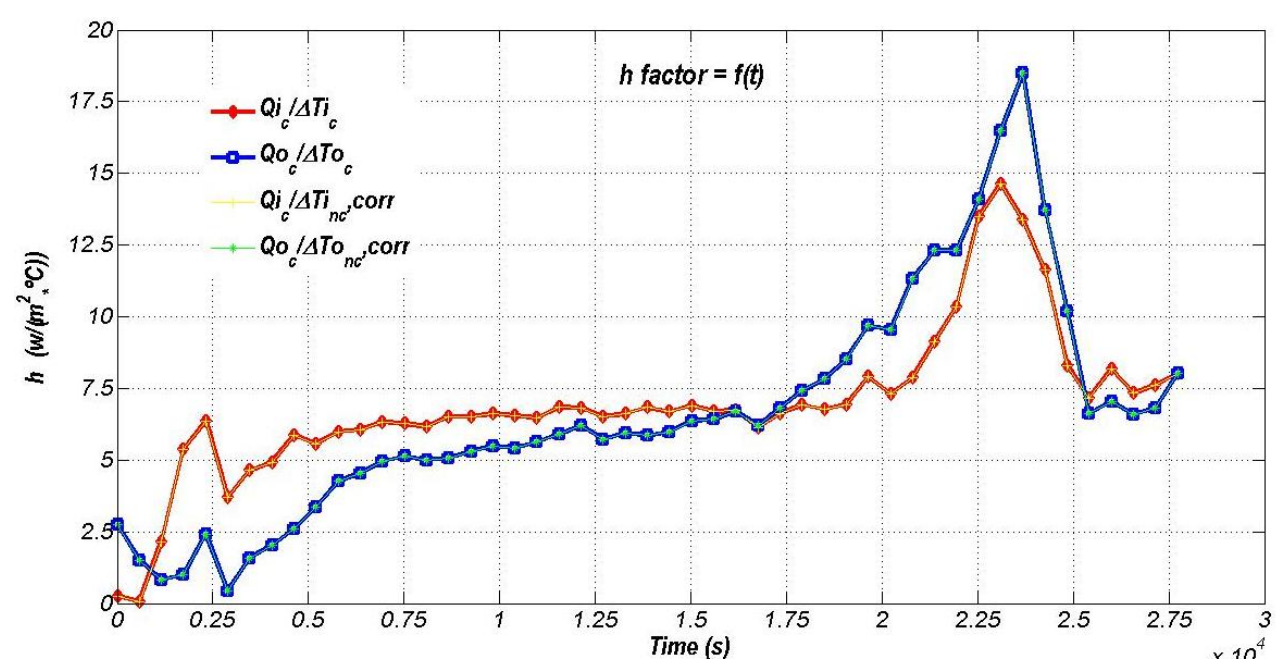

Fig 9 Instantaneous proportionality factor as a function of time 
The behavior of heat fluxes of some pixels as a function of time can be seen in the Fig 10. This figure compares the released heat captured by contact sensors $\boldsymbol{Q}_{c}$ and the behavior of calibrated heat flux of some pixels by thermal camera during exothermal heat process.

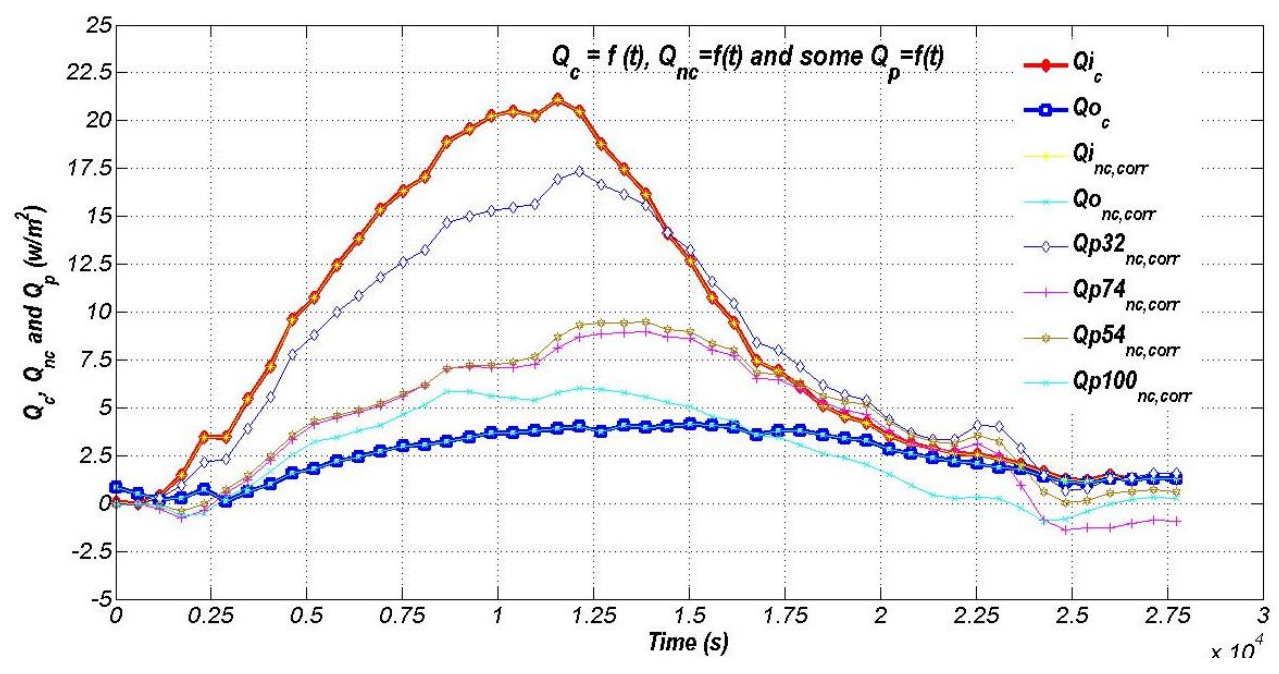

Fig 10 Contact and some pixel heat fluxes along the mould

\section{Conclusions and future works}

Through the present work demonstrates that by means of an in-situ calibration experimental method is possible to measure the two-dimensional heat flux by non-intrusive techniques, i.e. thermal camera, using as calibration reference accuracy zero-dimensional sensors. This measurement method has been applied in order to append new technical ideas for improvement of the manufacturing composite processes, especially for Resin Infusion (RI). This method offers great flexibility and versatility, as well as capability of area monitoring to measure heat flux for any location on the mould, at the same time instant. This overcomes the typical constraints of contact thermal sensors about dimensionality, geometry shape, size, and fixed location of them. Therefore, this experimental measurement method based on a proper combination of contact and non-contact sensors is very interesting for the industry, since it might extended to monitor the curing behavior of very large composite parts. In general, this heat flux calibration method might achieve higher accuracy knowing the amount of active mass (resin) locally concentrated along the mould. This can be obtained by previous characterizations regarding different thicknesses with different fiber volume fraction. In this sense, we are developing some work.

The implementation of these information systems in real time should improve the attractiveness for low cost techniques, as far as it is verified that the mechanical properties are improved by obtaining controlled homogeneous cured in the mould as well as to predict thicknesses for the finished part. In the future, the mechanical performance tests on the finished part should be analyzed to investigate the effect of inhomogeneous polymerization along the plates and the result should show varying mechanical properties for different areas along the same part. This item could become a future work as the real-time computation for the degree of cure DoC along several zones of mould using the calibrated measurement of heat. So far, the accuracy of kinetic models have been validated experimentally only by zero-dimensional sensor which induces uncertainty and ignorance about cure wherever the mould. In this way, future works it also could aim to evaluate the curing kinetics of the mould directly as in some research where they already attempted do it by thermocouples. Moreover, the predictions and the accuracy of kinetic models according to DSC previous characterizations could be validated experimentally by means of the experimental approach presented here.

\section{Acknowledgements}

The authors acknowledge the financing support provided by UCH CEU - University Cardenal Herrera (Valencia Spain) and the support at the experimentation by Chair of High Performance Composite (Montreal, Canada)

\section{REFERENCES}

[1] N. C. Correia, F. Robitaille, A. C. Long, C. D. Rudd, Simacek, and S. G. Advani, "Analysis of the vacuum infusion moulding process: I. Analytical formulation," Composites Part A: Applied Science and Manufacturing, vol. 36, no. 12, pp. 1645-1656, Dec.2005. 
[2] N. Montés, F. Sánchez, and A.Falco, "A topological approach for the flow behaviour characterization in LCM processes," Twente (Netherland): 2009.

[3] U.Pineda, N.Montés, L.Domenech, and F.Sánchez, "On-line Measurement of the resin infusion flow variables using artificial vision technologies,", 1 ed 2010.

[4] Joseph D.Menczel, Lawrence Judovits, R.Bruce Prime, Harvey E.Bair, Mike Reading, and Steven Swier, "Differential Scanning Calorimetry (DSC)," in Thermal Analysis of Polymers: Fundamentals and Aplications. Joseph D.Menczel and R.Bruce Prime, Eds. Wiley, 2009, pp. 7-239.

[5] P. Wang, S. Drapier, J. Molimard, A. Vautrin, and J. C. Minni, "Characterization of Liquid Resin Infusion (LRI) filling by fringe pattern projection and in situ thermocouples," Composites Part A: Applied Science and Manufacturing, vol. 41, no. 1, pp. 36-44, Sept.2009.

[6] E. Ruiz and F. Trochu, "Thermomechanical Properties during Cure of Glass-Polyester RTM Composites: Elastic and Viscoelastic Modeling," Journal of Composite Materials, pp. 881-916, May2005.

[7] M. J. Perry, L. J. Lee, and C. W. Lee, "On-Line Cure Monitoring of Epoxy/Graphite Composites Using a Scaling Analysis and a Dual Heat Flux Sensor," Journal of Composite Materials, vol. 26, no. 2, pp. 274-292, Jan.1992.

[8] K. T. Hsiao, R. Little, O. Restrepo, and B. Minaie, "A study of direct cure kinetics characterization during liquid composite molding," Composites Part A: Applied Science and Manufacturing, vol. 37, no. 6, pp. 925-933, June2006.

[9] A. Cusano, G. Breglio, M. Giordano, A. Calabrò, A. Cutolo, and L. Nicolais, "An optoelectronic sensor for cure monitoring in thermoset-based composites," Sensors and Actuators A: Physical, vol. 84, no. 3, pp. 270-275, Sept.2000.

[10] Dara L.Woerdeman, Julie K.Spoerre, Kathleen M.Flynn, and Richard S.Parnas, "Cure monitoring of the liquid composite molding process using fiber optic sensors," Polymer Composites, vol. 18, no. 1, pp. 133-150, 1997.

[11] C. Li, M. Cao, R. Wang, Z. Wang, Y. Qiao, L. Wan, Q. Tian, H. Liu, D. Zhang, T. Liang, and C. Tang, "Fiber-optic composite cure sensor: monitoring the curing process of composite material based on intensity modulation," Composites Science and Technology, vol. 63, no. 12, pp. 1749-1758, Sept.2003.

[12] Stephen D.Senturia and Norman F.Shepapard, "Dielectric Analysis of Thermoset Cure," Advances in Polymer Science, vol. 80, pp. 1-47, 1986.

[13] A. Mcllhagger, D. Brown, and B. Hill, "The development of a dielectric system for the on-line cure monitoring of the resin transfer moulding process," Composites Part A: Applied Science and Manufacturing, vol. 31, no. 12, pp. 1373-1381, Dec.2000.

[14] S. Comas-cardona, S. Ziaee, and S. G. Advani, "Spatially homogeneous gelation in liquid composite molding," Polym Eng Sci, vol. 42, no. 8, pp. 1667-1673, 2002.

[15] J. Dorighi, S. Krishnaswamy, and J. Achenbach, "A Fiber Optic Ultrasonic System to Monitor the Cure of Epoxy," Research in Nondestructive Evaluation, vol. 9, no. 1, pp. 13-24, Sept.1997.

[16] E. Schmachtenberg, J. Schulte zur Heide, and J. Töpker, "Application of ultrasonics for the process control of Resin Transfer Moulding (RTM)," Polymer Testing, vol. 24, no. 3, pp. 330-338, May2005.

[17] P. A. Fomitchov, Y. K. Kim, A. K. Kromine, and S. Krishnaswamy, "Laser Ultrasonic Array System for Real-Time Cure Monitoring of Polymer-Matrix Composites," Journal of Composite Materials, vol. 36, no. 15, pp. 1889-1901, Aug.2002.

[18] MAPE Engineering Swithzerland S.A., "Infusion composite parts- Thermoflux technologies," 2007.

[19] J. H. Chen, A. Johnston, L. Petrescue, and M. Hojjati, "A novel calorimetry technique for monitoring electron beam curing of polymer resins," Radiation Physics and Chemistry, vol. 75, no. 2, pp. 336-349, Feb.2006.

[20] V. Sobotka and D. Delaunay, "Analysis and Control of Heat Transfer in an Industrial Composite Mold in RTM Polyester Automotive Process," Journal of Reinforced Plastics and Composites, vol. 26, no. 9, pp. 881-901, June2007.

[21] N. Boyard, M. Vayer, C. Sinturel, R. Erre, and D. Delaunay, "Modeling PVTX diagrams: Application to various blends based on unsaturated polyester: Influence of thermoplastic additive, fillers, and reinforcements," J. Appl. Polym. Sci., vol. 92, no. 5, pp. 2976-2988, 2004.

[22] F. Lionetto, A. Tarzia, and A. Maffezzoli, "Air-Coupled Ultrasound: A Novel Technique for Monitoring the Curing of Thermosetting Matrices," Ultrasonics, Ferroelectrics and Frequency Control, IEEE Transactions on, vol. 54, no. 7, pp. 1437-1444, July2007.

[23] Thomas J.Walsh, "Resin Cure Monitor," Houston, Tex (USA) Patent 6,099,162, Aug.8, 2000.

[24] U.Pineda, N.Montés, F.Sánchez, and L.Domenech, "Experimental Evaluation of Heat Transfer Measurements during LCM Processes by Intrusive and Non-Intrusive Heat Flux Sensors," 18th International Conference on Composite Materials, 2011.

[25] M.Ochs, T.Horbach, A.Schulz, R.Koch, and H-J.Bauer, "A novel calibration method for an infrared thermography system applied to heat transfer experiments," Measurement Science and Technology, vol. 20, no. (2009) 075103, pp. 1-9, 2009.

[26] C. Fuentes, M. Liniers, J. Guasp, J. Doncel, J. Botija, G. Wolfers, J. Alonso, M. Acedo, E. Sanchez, G. Marcon, M. Weber, R. Carrasco, X. Sarasola, B. Zurro, and J. Tera, "Thermographic calorimetry of the neutral beam injectors heating beams at TJ-II," Review of Scientific Instruments, vol. 77, no. 10, pp. 10E519-10E519-3, Oct.2006.

[27] G. Carlomagno and G. Cardone, "Infrared Thermography for convective heat transfer measurements," Experiments in Fluids, vol. 49, no. 12010.

[28] NEC, "Advanced Thermo - TVS500EXZ Manual Rev1.0," Tokyo, Japan: NEC Avio Infrared Technologies Co., Ltd, 2008, pp. 1-144. 
[29] Gerald C.Holst, Common Sense Approach to Thermal Imaging JDC Publishing and SPIE Optical Engineering Press, 2000.

[30] Vered Scharf and Abrahm Katzir, "Four-band fiber optic radiometry for true temperature measurements during exothermal process," Optical Engineering, vol. 41, no. 7, pp. 1502-1506, Jan.2002.

[31] Frank Liebmann, "Emissivity - The crux of accurate radiometric measurement," Fluke Corporation, 2006.

[32] J.M Bullin, "Convective Heat Transfer and Infrared Thermography (IRTh)," Journal of Applied Fluid Mechanics, vol. 3, no. 1, pp. 55-62, 2010. 